\title{
Hemorrhage associated with ventriculoperitoneal shunt placement in aneurysmal subarachnoid hemorrhage patients on a regimen of dual antiplatelet therapy: a retrospective analysis
}

\author{
Joseph S. Hudson, BA, ${ }^{1}$ Yasunori Nagahama, MD, ${ }^{1}$ Daichi Nakagawa, MD, ${ }^{1}$ \\ Robert M. Starke, MD, MSc, ${ }^{6}$ Brian J. Dlouhy, MD, ${ }^{1}$ James C. Torner, PhD, MS, ${ }^{2}$ \\ Pascal Jabbour, MD, ${ }^{3}$ Lauren Allan, DO, ${ }^{4}$ Colin P. Derdeyn, MD, ${ }^{5}$ Jeremy D. W. Greenlee, MD, ${ }^{1}$ and \\ David Hasan, MD'
}

Departments of ${ }^{1}$ Neurological Surgery and ${ }^{5}$ Radiology, University of lowa Hospitals and Clinics, lowa City; ${ }^{2}$ Department of Epidemiology, University of lowa College of Public Health, lowa City; ${ }^{4}$ Department of General Surgery, Mercy Medical Center, Des Moines, lowa; ${ }^{3}$ Department of Neurological Surgery, Thomas Jefferson University Hospital, Philadelphia, Pennsylvania; and ${ }^{6}$ Departments of Neurological Surgery and Radiology, University of Miami Hospital, Miami, Florida

OBJECTIVE Intracranial stenting and flow diversion require the use of dual antiplatelet therapy (DAPT) to prevent instent thrombosis. DAPT may significantly increase the risk of hemorrhagic complications in patients who require subsequent surgical interventions. In this study, the authors sought to investigate whether DAPT is a risk factor for hemorrhagic complications associated with ventriculoperitoneal (VP) shunt placement in patients with aneurysmal subarachnoid hemorrhage (aSAH). Moreover, the authors sought to compare VP shunt complication rates with respect to the shunt's location from the initial external ventricular drain (EVD) site.

METHODS Patients with aSAH who presented to the authors' institution from July 2009 through November 2016 and required VP shunt placement for persistent hydrocephalus were included. The rates of hemorrhagic complications associated with VP shunt placement were compared between patients who were on a regimen of DAPT (aspirin and clopidogrel) for use of a stent or flow diverter, and patients who underwent microsurgical clipping or coiling only and were not on DAPT using a backward stepwise multivariate analysis. Rates of radiographic hemorrhage and infection-related VP shunt revision were compared between patients who underwent VP shunt placement along the same track and those who underwent VP shunt placement at a different site (contralateral or posterior) from the initial EVD.

RESULTS A total of 443 patients were admitted for the management of aSAH. Eighty of these patients eventually required VP shunt placement. Thirty-two patients $(40 \%)$ had been treated with stent-assisted coiling or flow diverters and required DAPT, whereas 48 patients $(60 \%)$ had been treated with coiling without stents or surgical clipping and were not on DAPT at the time of VP shunt placement. A total of 8 cases $(10 \%)$ of new hemorrhage were observed along the intracranial proximal catheter of the VP shunt. Seven of these hemorrhages were observed in patients on DAPT, and 1 occurred in a patient not on DAPT. After multivariate analysis, only DAPT was significantly associated with hemorrhage (OR 31.23, 95\% Cl 2.98-327.32; $p=0.0001$ ). One patient (3\%) on DAPT who experienced hemorrhage required shunt revision for hemorrhage-associated proximal catheter blockage. The remaining 7 hemorrhages were clinically insignificant. The difference in rates of hemorrhage between shunt placement along the same track and placement at a different site of 0.07 was not significant $(6 / 47$ vs $2 / 32, p=0.46)$. The difference in infection-related VP shunt revision rate was not significantly different ( $1 / 47 \mathrm{vs} 3 / 32, p=0.2978)$.

CONCLUSIONS This clinical series confirms that, in patients with ruptured aneurysms who are candidates for stentassisted coiling or flow diversion, the risk of clinically significant VP shunt-associated hemorrhage with DAPT is low. In an era of evolving endovascular therapeutics, stenting or flow diversion is a viable option in select aSAH patients. https://thejns.org/doi/abs/10.3171/2017.5.JNS17642

KEY WORDS subarachnoid hemorrhage; dual antiplatelet therapy; ventriculoperitoneal shunt; aspirin; clopidogrel; posthemorrhagic hydrocephalus; complications; chief resident; vascular disorders

ABBREVIATIONS aSAH = aneurysmal subarachnoid hemorrhage; DAPT = dual antiplatelet therapy; EVD = external ventricular drain; VP = ventriculoperitoneal. SUBMITTED March 11, 2017. ACCEPTED May 23, 2017.

INCLUDE WHEN CITING Published online November 10, 2017; DOI: 10.3171/2017.5.JNS17642. 
A PPROXIMATELY 30,000 people experience an aneurysmal subarachnoid hemorrhage (aSAH) in the United States every year, with an estimated mortality rate of $25 \% .^{7,8,16}$ Approximately one-third of all stroke-related years of potential life lost before the age of 65 years can be attributed to aSAH. ${ }^{8}$ Reductions in time to surgery coupled with the evolution of endovascular treatment have improved outcomes in aSAH patients. Despite these advances, comorbid conditions such as posthemorrhagic hydrocephalus continue to influence long-term outcomes in those who survive the initial hemorrhage. The majority of aSAH patients present with hydrocephalus and require ventriculostomy placement. An estimated $10 \%-20 \%$ of aSAH patients eventually require placement of a ventriculoperitoneal (VP) shunt for permanent diversion of CSF due to posthemorrhagic hydrocephalus. ${ }^{6,12,14}$ Historically, VP shunt placement has been associated with low rates of hemorrhagic complication. ${ }^{12}$

The development of novel endovascular stents and flow diverters has expanded the utility of endovascular therapy, enabling wide-necked and complex aneurysms to be treated without open microsurgery. As a result, stents and flow diverters are being used with increasing frequency in the setting of aSAH. However, the use of stents or flow diverters requires dual antiplatelet therapy (DAPT) to prevent in-stent thrombosis. This creates a trade-off, requiring physicians to balance the widening applicability of these techniques with the potential risk of procedure-related hemorrhage in patients who might eventually require additional surgery.

An earlier study conducted at our institution demonstrated that the rate of ventriculostomy-related hemorrhagic complication was higher in aSAH patients treated with stent-assisted coiling and DAPT than those treated without a stent. ${ }^{10}$ Moreover, our group's subsequent study involving 37 patients revealed a low but increased rate of symptomatic intracranial hemorrhage associated with placement of a VP shunt in patients on a regimen of DAPT for stent-assisted coiling..$^{13}$ These small studies suggest that an elevated risk of hemorrhagic complication exists in these patients.

In this study, involving a much larger number of patients over a period of 6.5 years, we sought to investigate whether DAPT required for use of intracranial stents or flow diverters is a risk factor for hemorrhagic complication from VP shunt placement in aSAH patients. We secondarily aimed to identify whether placement of VP shunts using the same track or a different site with respect to the initial external ventricular drain (EVD) influences the rate of both VP shunt-associated hemorrhagic complication and infection-associated VP shunt revision. Lastly, in a number of recent studies, resident involvement in neurosurgical care has not been found to impact patient outcomes. ${ }^{3,11,17}$ We aimed to qualitatively assess the number of VP shunt-associated hemorrhagic complications with respect to chief resident coverage periods to assess safety of resident involvement for this patient subset.

\section{Methods}

\section{Study Population and Procedural Technique}

The study was approved by the University of Iowa Hospitals and Clinics Institutional Review Board. Patient data were collected and entered prospectively into a quality control database. Data were collected from patients with aSAH presenting to the University of Iowa Hospitals and Clinics between July 2009 and November 2016. Our study included patients from our prior analysis..$^{13}$ All available medical records of aSAH patients who underwent VP shunt placement during this period were retrospectively reviewed. Baseline characteristics for the entire study population were collected at the time of admission. These characteristics are summarized in Table 1.

The techniques used for placement of the VP shunts have been previously described.$^{13}$ All aSAH patients who initially required a ventriculostomy but who were not able to tolerate EVD weaning underwent placement of a VP shunt at a later time during initial hospitalization, typically 7-21 days after admission. The EVD was removed prior to shunt placement and was never connected to the shunt system. The standard technique for shunt placement was employed. VP shunts were placed via the frontal approach with a bur hole at Kocher's point or via the posterior approach, as previously described. ${ }^{13}$ For VP shunts placed via the frontal approach, the decision to use the side ipsilateral or contralateral to the ventriculostomy site was made clinically in order to minimize infection risk (e.g., history of CSF leak). Given the elevated risk of complication in aSAH patients, all procedures were performed by senior-level trainees (i.e., chief residents) under the supervision of a faculty vascular neurosurgeon. For patients who underwent endovascular interventions involving use of a stent or flow diverter, DAPT was administered as previously described by Mahaney et al. ${ }^{13}$ and was not discontinued during the perioperative period for VP shunt placement. These patients were given $600 \mathrm{mg}$ clopidogrel and $325 \mathrm{mg}$ aspirin during the initial endovascular procedure. Postoperatively, they were maintained on a regimen of 325 $\mathrm{mg}$ aspirin daily and $75 \mathrm{mg}$ clopidogrel daily.

\section{Determination of Hemorrhagic Complications}

The postoperative CT scans obtained in each patient

TABLE 1. Baseline demographic characteristics of the study population

\begin{tabular}{lcc}
\hline \multicolumn{1}{c}{ Characteristic } & $\begin{array}{c}\text { Patients w/ } \\
\text { VP Shunt \& } \\
\text { DAPT }\end{array}$ & $\begin{array}{c}\text { Patients w/ } \\
\text { VP Shunt \& } \\
\text { No DAPT }\end{array}$ \\
\hline Mean age, yrs (SD) & $59.45(12.26)$ & $54.45(12.81)$ \\
\hline No. of patients & 32 & 48 \\
\hline Male & $12(15 \%)$ & $15(18.7 \%)$ \\
\hline Female & $20(25 \%)$ & $33(41.3 \%)$ \\
\hline Mean follow-up time, yrs (SD) & $2.40(1.98)$ & $2.19(2.00)$ \\
\hline Mean H\&H grade at presentation (SD) & $3.32(0.91)$ & $3.21(1.05)$ \\
\hline Mean Fisher grade at presentation (SD) & $3.41(0.80)$ & $3.5(0.74)$ \\
\hline No. of VP shunts stratified by site from & & \\
1st EVD* & & \\
\hline$\quad$ Same & $18(22.5 \%)$ & $29(36.3 \%)$ \\
\hline$\quad$ Different & $14(17.5 \%)$ & $18(22.5 \%)$ \\
\hline H\&H = Hunt and Hess. & & \\
$*$ One patient did not receive an initial EVD. & &
\end{tabular}


were reviewed by an attending neuroradiologist who was not involved in the study at the University of Iowa Hospitals and Clinics for clinical purposes. Identified hemorrhages were further reviewed independently by 2 attending neurosurgeons not familiar with details of the cases (i.e., whether patients were on DAPT) who analyzed the CT scans for hemorrhage location and extent. Intracranial hemorrhages were deemed VP shunt-related if they occurred along the track of the ventricular catheter. A hemorrhage that occurred at a site remote from the catheter was judged to not be VP shunt-related. The hemorrhages were judged to be clinically significant, as per our prior study, ${ }^{13}$ if they were temporally associated with observed declines in neurological status, required further surgical interventions, or caused seizures in the patient. If the observed hemorrhages were judged to be not clinically significant, they were categorized as radiographic hemorrhages. The same neurosurgeons also independently predicted whether those hemorrhages would cause clinical symptoms based only on CT scanning findings.

\section{Statistical Analysis and Temporal Organization}

The odds ratios for hemorrhage with respect to DAPT usage, age, sex, VP shunt approach, ventriculostomy approach, Hunt and Hess grade on presentation, Fisher grade on presentation, World Federation of Neurosurgical Societies grade on presentation, and follow-up time were analyzed using logistic regression. Variables associated with hemorrhage in univariate analyses $(p<0.3)$ were included in a backward, stepwise, multivariate analysis. Rates of hemorrhage and revision due to associated infection with respect to VP shunt location were compared using the Fisher exact test. Differences were calculated with $90 \%$ confidence intervals. Cases of postoperative intracranial hemorrhage along the VP shunt catheter were temporally organized into 6-month time blocks, approximating an individual chief resident's coverage of cerebrovascular cases, and plotted for qualitative review.

\section{Results}

Between July 2009 and November 2016, 443 patients with aSAH were admitted to the University of Iowa Hospitals and Clinics. Of these patients, 80 eventually required VP shunt placement for the treatment of persistent hydrocephalus. The demographic characteristics of these patients are summarized in Table 1 . Of these 80 patients requiring a VP shunt, $32(40 \%)$ had been treated with stentassisted coiling or flow diverters and required DAPT. The remaining 48 patients $(60 \%)$ had been treated with coiling without stents or surgical clipping, and were not on DAPT at the time of VP shunt placement.

Eight cases (10\%) of new intracranial hemorrhage along the ventricular catheter of the VP shunt were observed (Fig. 1). Seven of these hemorrhages were observed in patients on DAPT (22\%) (Fig. 1A-G). One new radiographic intracranial hemorrhage occurred along the shunt track in a patient who underwent surgical clipping and was not on DAPT (Fig. 1H). After multivariate analysis, only DAPT was significantly associated with hemorrhage (OR $31.23,95 \%$ CI 2.98-327.32; p = 0.0001) (Table 2). Of the 7 hemorrhages that occurred in patients on DAPT, only 1 hemorrhage $(1 / 32,3 \%)$ was clinically significant, requiring
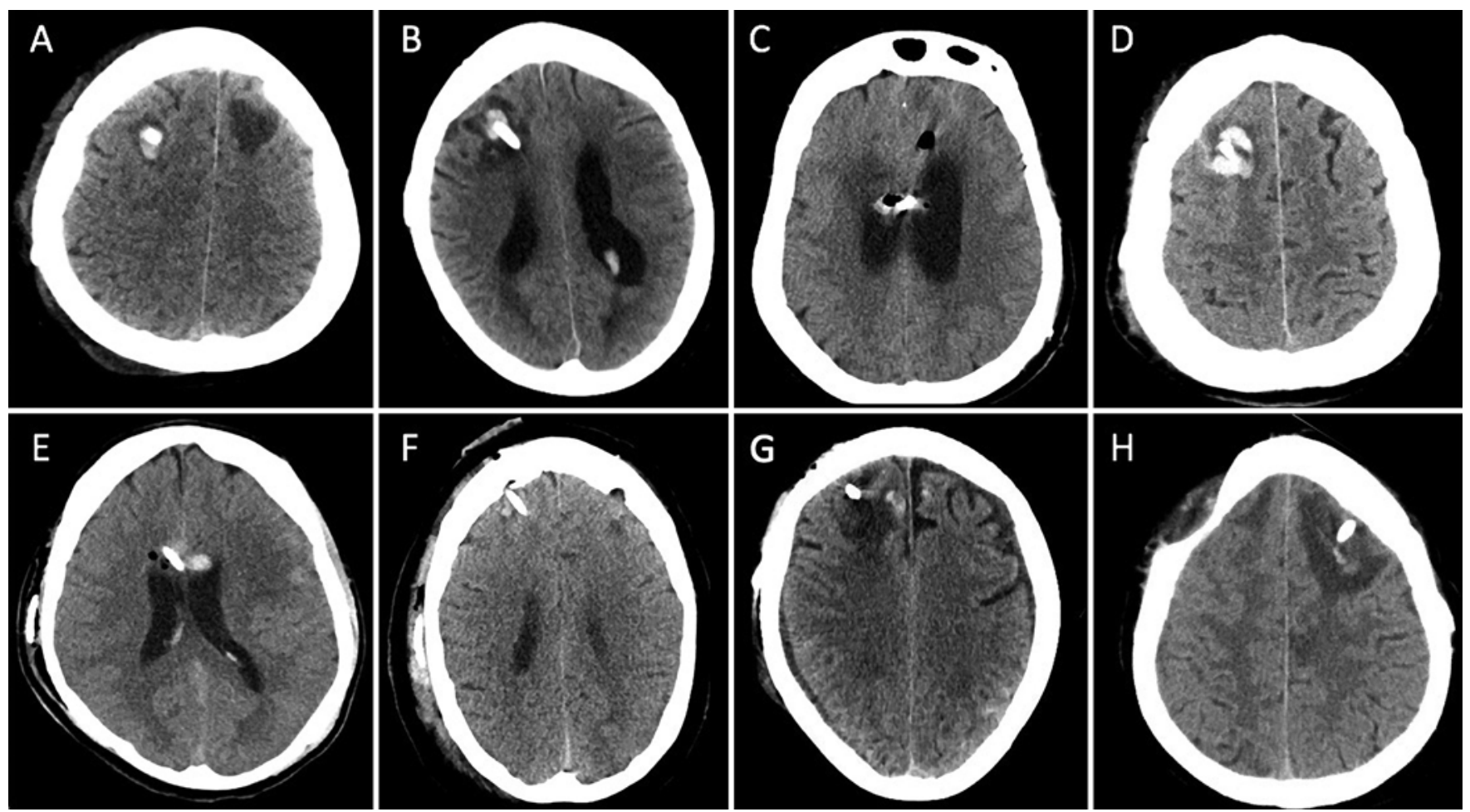

FIG. 1. Representative CT brain images of all study patients with identified hemorrhage associated with VP shunt placement. A-G: Images obtained in the 7 patients on DAPT. H: Image obtained in the patient not on DAPT. 
TABLE 2. Univariate and multivariate predictors of ventriculoperitoneal shunt-associated hemorrhage

\begin{tabular}{|c|c|c|c|c|c|c|}
\hline \multirow[b]{2}{*}{ Variable } & \multicolumn{3}{|c|}{ Univariate } & \multicolumn{3}{|c|}{ Multivariate } \\
\hline & OR & $95 \% \mathrm{Cl}$ & $p$ Value & OR & $95 \% \mathrm{Cl}$ & p Value \\
\hline Age & 1.01 & 0.94 to 1.07 & 0.87 & & & \\
\hline Sex (female) & 0.39 & 0.05 to 3.16 & 0.38 & 0.33 & 0.05 to 1.86 & 0.21 \\
\hline VP shunt approach (rt) & 0.39 & 0.04 to 4.15 & 0.43 & & & \\
\hline Ventriculostomy approach (rt) & 0.84 & 0.06 to 11.29 & 0.90 & & & \\
\hline Same-sided approach as EVD & 9.84 & 0.96 to 101.00 & 0.0008 & 4.63 & 0.71 to 30.25 & 0.11 \\
\hline DAPT & 31.58 & 2.03 to 491.31 & 0.033 & 31.23 & 2.98 to 327.32 & 0.0001 \\
\hline $\mathrm{H} \& \mathrm{H}$ grade & 0.51 & 0.06 to 4.20 & 0.53 & & & \\
\hline Fisher grade & 1.15 & 0.28 to 4.72 & 0.84 & & & \\
\hline WFNS grade & 1.87 & 0.56 to 6.24 & 0.28 & 1.57 & 0.77 to 3.21 & 0.22 \\
\hline Follow-up time & 1.22 & -0.76 to 1.96 & 0.41 & & & \\
\hline
\end{tabular}

WFNS $=$ World Federation of Neurosurgical Societies.

VP shunt revision due to a hemorrhage-associated proximal blockage (Fig. 1C). This patient required an additional shunt revision for infection and poor wound healing during a later hospital admission, but there was no further hemorrhage-related permanent morbidity. All observed hemorrhages were judged to be around the track of the ventricular catheter, as determined separately by 2 attending neurosurgeons. In 3 of the hemorrhage cases, a very small amount of blood was noted in the occipital horn of the lateral ventricles, likely representing tracking of the blood along the catheter into the ventricles. One hemorrhage was judged to be greater than $2 \mathrm{~cm}$ (Fig. 1E). All observed hemorrhages were deemed likely to be asymptomatic based on imaging alone. One hemorrhage occurred along a previously placed VP shunt ventricular catheter track (Fig. 1G). No hemorrhages occurred in individuals receiving a VP shunt with a posterior-approach shunt placement.

In the 8 patients with observed hemorrhage, 6 cases occurred with placement of the VP shunt ventricular catheter using the same track as the prior EVD, while 2 cases occurred in patients with VP shunt placement at a different site. The difference in observed hemorrhage between these 2 groups was 0.07 (90\% CI $0.06-0.21 ; 6 / 47$ vs $2 / 32$; $\mathrm{p}=0.46$ ). There were 4 cases of VP shunt revision due to infection. One case occurred in a patient who underwent VP shunt placement using the same track as the prior EVD, while 3 cases occurred with VP shunt placement at a different site. The difference in infection rate of 0.072 (90\% CI -0.034 to 0.212 ) was not statistically significant between these 2 groups $(1 / 47$ vs $3 / 32 ; p=0.2978)$. The average follow-up time for patients with infection-related shunt revision was 2.8 years. Eight cases of postoperative intracranial hemorrhage along the VP shunt catheter were temporally organized into 6-month blocks and plotted. The results are shown in (Fig. 2). Two blocks were associated with 2 cases each of VP shunt-associated hemorrhage. No more than 2 cases of hemorrhage were observed during any individual 6-month coverage period.

\section{Discussion}

In the setting of acutely ruptured intracranial aneurysms, there is a reluctance to use intracranial stents or flow diverters because DAPT is subsequently required to prevent in-stent thrombosis. The possibility of additional surgical maneuvers underlies this avoidance. However, the usage of stents or flow diverters may be favorable in some situations. In fact, a number of studies have suggested that stents and flow diverters may be used effectively to treat ruptured aneurysms when other therapeutic modalities are less clinically appealing., ${ }^{1,2,4,15}$ Although the risk profile was found to be acceptable in these analyses, the possibility of iatrogenic hemorrhage secondary to DAPT was continually emphasized. ${ }^{1,2,4,15}$

We previously performed a small retrospective singleinstitution study of 37 patients undergoing placement of a

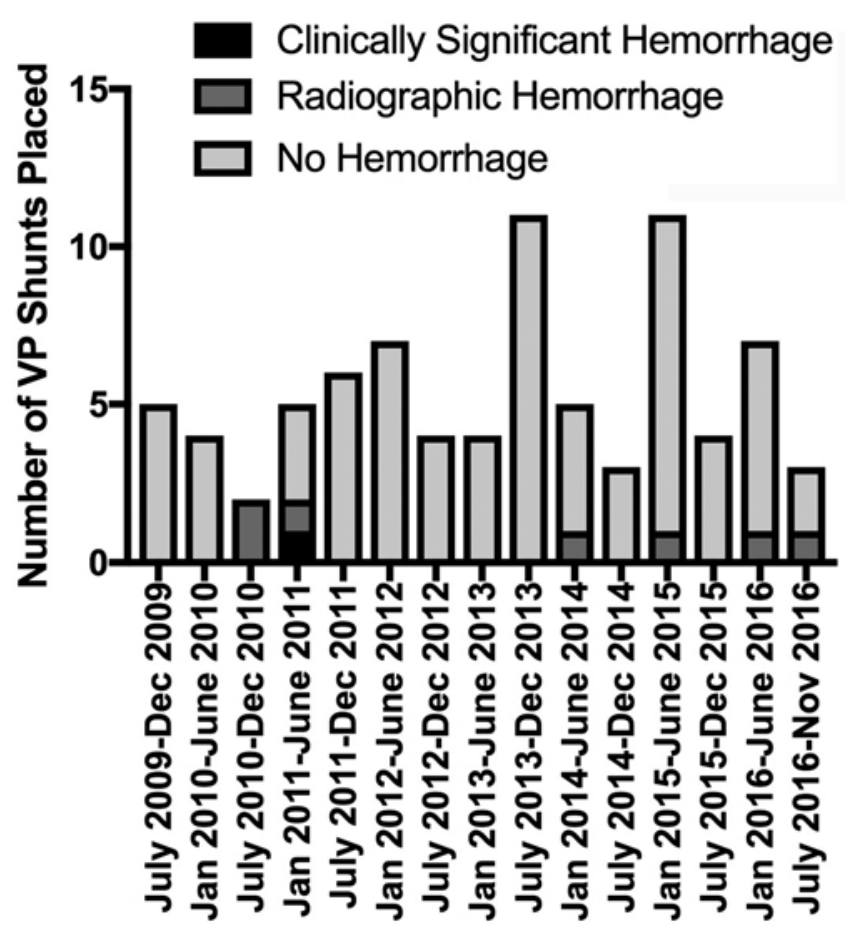

FIG. 2. Bar graph of VP shunt placements and associated hemorrhages divided into 6-month blocks corresponding to individual chief resident coverage periods. 
VP shunt after treatment for aSAH.${ }^{13}$ Four new VP shuntassociated intracranial hemorrhages were observed in patients receiving DAPT, while none occurred in those not receiving DAPT (difference 0.333, 90\% CI 0.093-0.607; $4 / 12$ vs $0 / 25 ; p=0.0075$ ). Only one of these 4 observed hemorrhages was deemed clinically significant, as it required shunt revision. In our current study, we sought to reevaluate our previous findings by analyzing a larger number of patients over an extended period of time. We found that the rate of observed hemorrhage was significantly higher in the 32 patients treated with DAPT. The hemorrhage rate in patients receiving DAPT (22\%) is higher than the $0 \%-8.3 \%$ rate of VP shunt-associated hemorrhage reported in the neurosurgical literature. ${ }^{9,12}$ Only one of these hemorrhages was clinically significant, requiring shunt revision, although the patient did not suffer permanent morbidity. These data confirm that continuation of DAPT for prevention of in-stent thrombosis is safe and thus important during placement of VP shunts for persistent hydrocephalus in aSAH patients.

Ultimately, a stent with inherent antithrombotic properties might allow for the discontinuation of long-term and perioperative DAPT. Most recently, Chiu et al. described the first case in which a patient with a ruptured dissecting aneurysm was treated using aspirin as the sole antiplatelet agent following use of a Pipeline Embolization Device with shield technology (PED-SHIELD, CovidienMedtronic). ${ }^{5}$ Such technology holds promise to alleviate the risk of iatrogenic bleeding following post-aneurysm treatment DAPT.

Our secondary objective in this study was to evaluate whether VP shunt site selection with respect to EVD placement was associated with increased risk for shuntassociated hemorrhage or infection-related shunt revision. Our data suggest that VP shunt site selection is not associated with an elevated risk of hemorrhage. Our data, although statistically insignificant, identify more revisions due to shunt infection when a different VP shunt site is selected. Based on these data, we recommend the use of clinical judgment when selecting a prior EVD tract versus a different site for VP shunt placement in this patient subpopulation. Finally, although our data do not support a statistical test of complication rate variability between these time periods, our qualitative results suggest that the overall hemorrhagic complication rate per 6-month period may be relatively stable, thus implying that chief residents may safely be involved in the care of patients requiring DAPT and VP shunt placement.

\section{Conclusions}

Our 6.5-year study confirms that in patients with ruptured aneurysms who are candidates for stent-assisted coiling or flow diversion, there is elevated risk for VP shunt-associated radiographic hemorrhage, but the risk of clinically significant hemorrhage is low. The significant risk of in-stent thrombosis without DAPT in these patients means that it is critical to continue DAPT during the perioperative time for VP shunt placement. In an era of rapidly evolving endovascular therapeutics, these data suggest that treatment requiring DAPT at a teaching hospital is a viable treatment option in select aSAH patients. Our study is limited by its retrospective nature, single-institution scope, and limited statistical power. Further analysis on the topic is needed.

\section{References}

1. Amenta PS, Dalyai RT, Kung D, Toporowski A, Chandela S, Hasan D, et al: Stent-assisted coiling of wide-necked aneurysms in the setting of acute subarachnoid hemorrhage: experience in 65 patients. Neurosurgery 70:1415-1429, 2012

2. Bodily KD, Cloft HJ, Lanzino G, Fiorella DJ, White PM, Kallmes DF: Stent-assisted coiling in acutely ruptured intracranial aneurysms: a qualitative, systematic review of the literature. AJNR Am J Neuroradiol 32:1232-1236, 2011

3. Bydon M, Abt NB, De la Garza-Ramos R, Macki M, Witham $\mathrm{TF}$, Gokaslan ZL, et al: Impact of resident participation on morbidity and mortality in neurosurgical procedures: an analysis of 16,098 patients. J Neurosurg 122:955-961, 2015

4. Chalouhi N, Jabbour P, Singhal S, Drueding R, Starke RM, Dalyai RT, et al: Stent-assisted coiling of intracranial aneurysms: predictors of complications, recanalization, and outcome in 508 cases. Stroke 44:1348-1353, 2013

5. Chiu AHY, Ramesh R, Wenderoth J, Davies M, Cheung A, et al: Use of aspirin as sole oral antiplatelet therapy in acute flow diversion for ruptured dissecting aneurysms. J Neurointerv Surg 9:e18, 2017

6. Hoh BL, Kleinhenz DT, Chi YY, Mocco J, Barker FG II: Incidence of ventricular shunt placement for hydrocephalus with clipping versus coiling for ruptured and unruptured cerebral aneurysms in the Nationwide Inpatient Sample database: 2002 to 2007. World Neurosurg 76:548-554, 2011

7. Ingall TJ, Whisnant JP, Wiebers DO, O'Fallon WM: Has there been a decline in subarachnoid hemorrhage mortality? Stroke 20:718-724, 1989

8. Johnston SC, Selvin S, Gress DR: The burden, trends, and demographics of mortality from subarachnoid hemorrhage. Neurology 50:1413-1418, 1998

9. Kamenova M, Croci D, Guzman R, Mariani L, Soleman $\mathrm{J}$ : Low-dose acetylsalicylic acid and bleeding risks with ventriculoperitoneal shunt placement. Neurosurg Focus 41(3):E4, 2016

10. Kung DK, Policeni BA, Capuano AW, Rossen JD, Jabbour PM, Torner JC, et al: Risk of ventriculostomy-related hemorrhage in patients with acutely ruptured aneurysms treated using stent-assisted coiling. J Neurosurg 114:1021-1027, 2011

11. Lim S, Parsa AT, Kim BD, Rosenow JM, Kim JY: Impact of resident involvement in neurosurgery: an analysis of 8748 patients from the 2011 American College of Surgeons National Surgical Quality Improvement Program database. J Neurosurg 122:962-970, 2015

12. Little AS, Zabramski JM, Peterson M, Goslar PW, Wait SD, Albuquerque FC, et al: Ventriculoperitoneal shunting after aneurysmal subarachnoid hemorrhage: analysis of the indications, complications, and outcome with a focus on patients with borderline ventriculomegaly. Neurosurgery 62:618627, 2008

13. Mahaney KB, Chalouhi N, Viljoen S, Smietana J, Kung DK, Jabbour P, et al: Risk of hemorrhagic complication associated with ventriculoperitoneal shunt placement in aneurysmal subarachnoid hemorrhage patients on dual antiplatelet therapy. J Neurosurg 119:937-942, 2013

14. O'Kelly CJ, Kulkarni AV, Austin PC, Urbach D, Wallace MC: Shunt-dependent hydrocephalus after aneurysmal subarachnoid hemorrhage: incidence, predictors, and revision rates. Clinical article. J Neurosurg 111:1029-1035, 2009

15. Peschillo S, Caporlingua A, Cannizzaro D, Resta M, Burdi $\mathrm{N}$, Valvassori L, et al: Flow diverter stent treatment for rup- 
tured basilar trunk perforator aneurysms. J Neurointerv Surg 8:190-196, 2016

16. Shea AM, Reed SD, Curtis LH, Alexander MJ, Villani JJ, Schulman KA: Characteristics of nontraumatic subarachnoid hemorrhage in the United States in 2003. Neurosurgery 61:1131-1138, 2007

17. Zygourakis CC, Lee J, Barba J, Lobo E, Lawton MT: Performing concurrent operations in academic vascular neurosurgery does not affect patient outcomes. J Neurosurg [epub ahead of print January 20, 2017. DOI: 10.3171/2016.6.JNS16822]

\section{Disclosures}

The authors report no conflict of interest concerning the materials or methods used in this study or the findings specified in this paper.

\section{Author Contributions}

Conception and design: Hasan, Hudson, Nagahama. Acquisition of data: Hudson, Dlouhy, Greenlee. Analysis and interpretation of data: Hasan, Hudson, Nakagawa, Derdeyn, Greenlee. Drafting the article: Hudson, Nagahama. Critically revising the article: all authors. Reviewed submitted version of manuscript: all authors. Approved the final version of the manuscript on behalf of all authors: Hasan. Statistical analysis: Hudson, Nakagawa, Starke, Torner. Study supervision: Hasan.

\section{Correspondence}

David Hasan, Department of Neurological Surgery, University of Iowa Hospitals and Clinics, 200 Hawkins Dr., Iowa City, IA 52242.email: david-hasan@uiowa.edu. 